\title{
Estrutura genética intrapopulacional em Cryptocarya moschata Nees (Lauraceae) $^{1}$
}

\author{
PEDRO L.R. DE MORAES ${ }^{2,5}$, REINALDO MONTEIRO ${ }^{3}$ e ROLAND VENCOVSKY ${ }^{4}$
}

(recebido: 28 de maio de 2003; aceito: 1 de abril de 2004)

\begin{abstract}
Intrapopulation genetic structure in Cryptocarya moschata Nees (Lauraceae)). Through the analysis of six enzyme systems (ACP, ALP, CAT, GOT, PPO and PO), with 18 polymorphic loci, allele frequencies of 24 alleles were calculated for 141 adult individuals of a natural population of Cryptocarya moschata (Brazilian nutmeg), from Carlos Botelho State Park (2403’21” S, 4759’36” W), São Miguel Arcanjo, SP, Brazil, which were sampled in an area ca. 647 ha. The spatial genetic structure was investigated by the spatial autocorrelation method, using Moran's $I$ coefficients estimated for 10 distance classes. Additionally, for the same distance classes, coefficients $r$ were calculated through a multivariate analysis. Both approaches were qualitatively similar, poiting out that 15 correlograms from Moran's $I$ coefficients were significant at $95 \%$ probability level. The total correlogram from $r$ coefficients indicated that there was a significant spatial structure for the distance class of 0 to $150 \mathrm{~m}$. By subdividing this class into $10 \mathrm{~m}$ intervals, positive spatial autocorrelation was detected in the majority of classes, suggesting that clusters of $C$. moschata trees, which were shorter than $50 \mathrm{~m}$ apart, were constituted of individuals with greater genetic similarity and were probably related. However, the greatest genetic similarity occurred among individuals being $105 \mathrm{~m}$ apart, which could be related to the kind of seed dispersal promoted by Brachyteles arachnoides (Primates - Cebidae). Results showed that for an adequate sampling, aiming to detect genetic diversity, the collection of individuals separated by a distance of $750 \mathrm{~m}$, or more, from each other, would be advisable.
\end{abstract}

Key words - Cryptocarya moschata, isozymes, Moran's I coefficients, multilocus analysis, spatial autocorrelation

RESUMO - (Estrutura genética intrapopulacional em Cryptocarya moschata Nees (Lauraceae)). Pela análise de seis sistemas enzimáticos (ACP, ALP, CAT, GOT, PPO e PO), com 18 locos polimórficos, foram calculadas as frequiências alélicas de 24 alelos referentes a 141 indivíduos adultos de uma população natural de Cryptocarya moschata (noz-moscada-do-Brasil) do Parque Estadual Carlos Botelho (2403'21" S, 4759'36” W), São Miguel Arcanjo, SP, amostrados em uma área de cerca de 647 ha. A estrutura genética espacial foi investigada através do método de autocorrelação espacial, utilizando-se coeficientes $I$ de Moran estimados para 10 classes de distância. Adicionalmente, para as mesmas classes de distância, estimaram-se os coeficientes $r$ através de uma análise multivariada. Ambas as abordagens mostraram-se similares qualitativamente, sendo que 15 correlogramas de coeficientes $I$ foram significativos ao nível de $95 \%$ de probabilidade. O correlograma total gerado pelos coeficientes $r$ indicou estruturação espacial significativa para a classe de distância de 0 a $150 \mathrm{~m}$. Pela subdivisão desta classe em intervalos de $10 \mathrm{~m}$, detectou-se autocorrelação espacial positiva na maioria das classes, indicando que os agrupamentos de árvores de C. moschata, que se encontram separadas a distâncias menores que $50 \mathrm{~m}$, são constituídos por indivíduos semelhantes geneticamente e provavelmente aparentados. Contudo, a maior similaridade genética ocorreu entre indivíduos distanciados em torno de $105 \mathrm{~m}$, podendo estar associada à dispersão de sementes promovida por Brachyteles arachnoides (Primates Cebidae). Pela análise dos resultados, conclui-se que para uma amostragem adequada, visando a detecção de diversidade genética, a coleta de indivíduos distanciados acima de $750 \mathrm{~m}$ seria recomendável.

Palavras-chave - Análise multilocos, autocorrelação espacial, coeficiente I de Moran, Cryptocarya moschata, isoenzimas

\section{Introdução}

A documentação na literatura sobre a dispersão espacialmente restrita, para um grande número de espécies vegetais, levou à predição de que as populações

\footnotetext{
1. Parte da tese de doutorado de P.L.R. de Moraes.

2. Universidade Estadual de Campinas, IB, Departamento de Botânica, Caixa Postal 6109, 13083-970 Campinas, SP, Brasil.

3. Universidade Estadual Paulista, IB, Departamento de Botânica, Caixa Postal 199, 13506-900, Rio Claro, SP, Brasil.

4. Escola Superior de Agronomia Luis de Queirós/USP, Departamento de Genética, Caixa Postal 83, 13418-900 Piracicaba, SP, Brasil.

5. Autor para correspondência: pmoraes@unicamp.br
}

de plantas estariam freqüentemente subdivididas geneticamente em uma escala local, como resultado da variabilidade espacial seletiva (heterogeneidade ambiental) ou da deriva genética local (isolamento pela distância) (Wright 1943, Levin \& Kerster 1974).

Os padrões espaciais e genéticos resultam geralmente da heterogeneidade ambiental e pressões seletivas diferenciais. Enquanto os padrões espaciais freqüentemente têm implicações genéticas, os padrões genéticos não aleatórios podem existir sem uma distribuição espacial não aleatória dos indivíduos. De forma contrária, uma população pode ter uma distribuição espacial não aleatória sem qualquer 
acompanhamento de estrutura genética (Loveless \& Hamrick 1984).

Segundo Hamrick (1990), os padrões de colonização e os eventos estocásticos que afetam o estabelecimento e a mortalidade também são importantes, juntamente com a heterogeneidade ambiental, para explicar a ocorrência agrupada das plantas em locais distintos. Vários trabalhos evidenciam que a variabilidade genética em populações de plantas está distribuída não aleatoriamente. Similarmente às próprias plantas, os genes e genótipos tendem a estar agrupados, com diferentes marcadores genéticos ocorrendo a pequenas distâncias.

Levins (1968) estabeleceu que espécies em ambientes de granulação grossa (pouca variação espaço-temporal $\approx$ estáveis) devem ter populações consistindo de mosaicos de ecótipos localmente adaptados. Como resultado, altos níveis relativos de variabilidade genética devem ser mantidos dentro das populações. Por outro lado, as espécies em ambientes de granulação fina (muito variáveis tanto no espaço, como no tempo) devem apresentar pouca adaptação local e são esperadas de manter uma menor variabilidade genética.

Segundo Lundkvist (1982), a diversidade genética entre os indivíduos é necessária para garantir uma capacidade de tamponamento da população contra flutuações temporais do ambiente. Adicionalmente, uma população de espécie arbórea florestal experiencia o ambiente como sendo de granulação grossa quando exposta às flutuações inter-anuais das condições locais, enquanto a heterogeneidade do espaço (situação de granulação fina) se manifesta na população no período de regeneração, quando um grande número de zigotos explora e utiliza os vários nichos em um ambiente heterogêneo.

Segundo Yeh (1989), a organização espacial de populações locais e seus padrões concomitantes de fluxo gênico são fatores importantes para uma espécie se tornar geneticamente diferenciada ao longo de sua amplitude geográfica. O padrão e a quantidade de pólen e de semente dispersados afetam o arranjo espacial dos genótipos. Um fluxo gênico limitado e seleção intrapopulacional em microhábitats podem promover a formação de agrupamentos familiares, entre os quais as frequiências alélicas podem diferir. Tal estrutura familiar é provavelmente uma característica comum de muitas populações de plantas, incluindo espécies arbóreas florestais (Linhart et al. 1981).

A autocorrelação espacial pode ser definida como a propriedade de variáveis aleatórias apresentarem valores para pares de locais, distanciados entre si, que são mais similares (autocorrelação positiva), ou menos similares (autocorrelação negativa) do que o esperado para pares de observações associados ao acaso (Legendre 1993).

Duas abordagens têm sido empregadas nas técnicas de autocorrelação espacial para a análise da variação genética espacial entre indivíduos diplóides de populações contínuas. Os genótipos individuais podem ser tratados como classes nominais, com suas distribuições espaciais descritas em termos de estatísticas de contagem de vizinhos pareados "join-counts" (Epperson \& Clegg 1986, Epperson \& Allard 1989, Schoen \& Latta 1989, Epperson 1995). De forma alternativa, os indivíduos podem ser caracterizados por suas freqüências alélicas, com a distribuição espacial dos alelos sendo descrita em termos de um coeficiente de autocorrelação, usualmente o coeficiente $I$ de Moran (Dewey \& Heywood 1988, Campbell \& Dooley 1992).

A maioria dos estudos empíricos tem analisado os padrões da variabilidade genética entre populações (Shapcott 1995, Telles et al. 2001). Contudo, os estudos dos padrões espaciais de variabilidade genética dentro de populações têm mostrado o poder da análise de autocorrelação espacial para descrever a estrutura genética e para detectar seleção natural (Sokal \& Oden 1978b, Epperson \& Clegg 1986, Dewey \& Heywood 1988, Epperson \& Allard 1989, Schoen \& Latta 1989, Knowles et al. 1992, Shea \& Furnier 2002).

Para a maioria das espécies arbóreas, a maior parte da diversidade gênica é constituída pela variação genética dentro das populações (Hamrick \& Godt 1989). A distribuição dessa variabilidade genética intrapopulacional reflete, ao menos em parte, a ação de processos genéticos em nível populacional, tais quais seleção e deriva genética. Esses processos, ou seja, a seleção para microhábitats e deriva genética devido ao fluxo gênico restrito, têm sido citados como as possíveis causas da distribuição não aleatória de genótipos em populações naturais (Loveless \& Hamrick 1984, Sokal et al. 1989, Epperson 1990).

De forma semelhante ao encontrado para a maioria das espécies arbóreas tropicais, Cryptocarya moschata apresenta a maior parte de sua variabilidade genética ocorrendo dentro das populações (Moraes et al. 1999, 2002). Com isto, torna-se natural perguntar como se organiza espacialmente a variabilidade genética em nível intrapopulacional.

No presente estudo, a análise de autocorrelação espacial foi empregada a partir dos dados isoenzimáticos de uma população de indivíduos adultos de Cryptocarya 
moschata, com o objetivo de verificar se a distribuição espacial dos genótipos possui forma aleatória ou estruturada. Para tanto, apresentam-se os resultados a partir dos coeficientes $I$ de Moran e dos coeficientes $r$ (multivariados) para diferentes classes de distância, discorrendo-se sobre as diferenças encontradas entre as duas abordagens.

\section{Material e métodos}

Foram amostrados 141 indivíduos adultos de Cryptocarya moschata Nees, da região norte do Parque Estadual Carlos Botelho (PECB; 2403'21" S e 47⒌'36" W), São Miguel Arcanjo, SP, que são os mesmos estudados por Moraes et al. $(1999,2002)$.

Na figura 1, apresenta-se a distribuição espacial dos indivíduos dentro da área de estudo de cerca de 647 ha, a partir de mapa georeferenciado, elaborado e descrito por Moraes (1997). A partir dos dados digitalizados, o programa IDRISI 4.1 (Eastman 1992) forneceu as coordenadas planas relativas de cada árvore, que foram utilizadas na análise de autocorrelação espacial.

A análise de autocorrelação espacial foi utilizada para investigar a estrutura genética espacial da população de
Cryptocarya moschata do PECB, empregando-se para tanto os genótipos individuais dos locos polimórficos de cada uma das 141 árvores adultas amostradas.

Dessa forma, cada alelo foi considerado como uma variável e codificado para cada árvore, da seguinte maneira: 1,0 para homozigotos, 0,5 para heterozigotos e 0,0 quando ausente. $\mathrm{Na}$ análise, foram utilizados os alelos que ocorreram em pelo menos seis indivíduos da população, pois os mais raros não proporcionariam nenhuma informação adicional a respeito da estrutura espacial. Para os locos dialélicos, somente um alelo foi analisado, uma vez que o segundo alelo apresenta distribuição dependente do primeiro.

Foram analisados 24 alelos, obtidos pela eletroforese em gel de amido, conforme metodologia descrita em Moraes et al. (1999, 2002), dos seguintes sistemas isoenzimáticos: fosfatase ácida (Acp-3 alelo 1), fosfatase alcalina (Alp-1 alelo 1, Alp-3 alelo 1, Alp-4 alelo 1, Alp-5 alelo 1), catalase (Cat-1 alelo 1, Cat-2 alelo 1, Cat-3 alelo 1, Cat-4 alelo 1), glutamatooxaloacetato transaminase (Got-1 alelos 1, 2 e 3, Got-2 alelos 1,2 e 3), polifenoloxidase (Ppo-4 alelo 1, Ppo-5 alelo 1), e peroxidase (Po-1 alelo 1, Po-2 alelo 1, Po-3 alelo 1, Po-4 alelos 1, 2 e 3 , e Po-5 alelo1).

A estrutura espacial da variação isoenzimática foi quantificada pelo coeficiente $I$ de Moran. Esse coeficiente quantifica a similaridade genética de pares de indivíduos

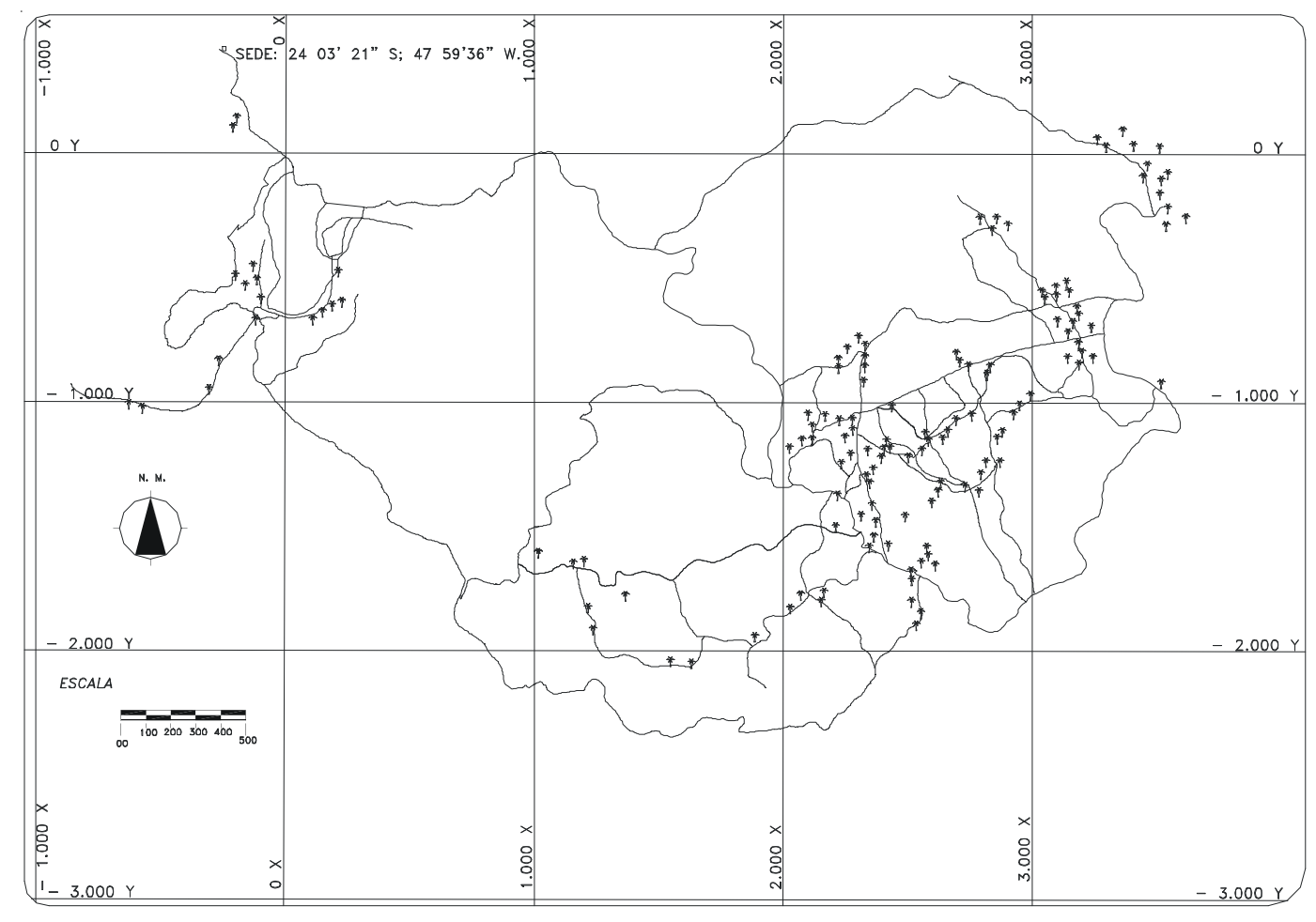

Figura 1. Mapa da área de estudo do P.E. Carlos Botelho, S. Miguel Arcanjo, SP, com localização das árvores amostradas de Cryptocarya moschata Nees.

Figure 1. Map of the study area at P.E. Carlos Botelho, S. Miguel Arcanjo, SP, with localization of sampled trees of Cryptocarya moschata Nees. 
adjacentes em relação à amostra da população como um todo. O coeficiente $I$ é calculado pela fórmula $\mathrm{I}=\left[\mathrm{n} \Sigma_{\mathrm{i} \neq \mathrm{j}} \mathrm{W}_{\mathrm{ij}}\left(\mathrm{x}_{\mathrm{i}}-\overline{\mathrm{x}}\right)\left(\mathrm{x}_{\mathrm{j}}-\overline{\mathrm{x}}\right)\right] /\left[\mathrm{W} \Sigma_{\mathrm{i}}\left(\mathrm{x}_{\mathrm{i}}-\overline{\mathrm{x}}\right)^{2}\right]($ Cliff \& Ord 1973), para dados categorizados e para um determinado alelo, sendo $\mathrm{n}=$ número de indivíduos utilizados na análise, $\mathrm{w}_{\mathrm{ij}}=$ pesos utilizados na comparação dos pontos, $\mathrm{x}_{\mathrm{i}}=$ valor dado ao indivíduo $\mathrm{i}\left(\mathrm{x}_{\mathrm{i}}=1 ; 0,5\right.$ ou 0$), \mathrm{x}_{\mathrm{j}}=$ valor dado ao indivíduo $\mathrm{j}\left(\mathrm{x}_{\mathrm{j}}=1 ; 0,5\right.$ ou 0$), \overline{\mathrm{x}}=$ valor médio obtido dos indivíduos analisados, e $\mathrm{W}=\Sigma_{\mathrm{ij}} \mathrm{W}_{\mathrm{ij}}$, a soma das entradas da matriz dos pesos (exceto para as entradas da diagonal).

$O$ coeficiente $I$ pode assumir valores entre +1 (autocorrelação positiva completa, ou seja, os indivíduos pareados são idênticos) e -1 (autocorrelação negativa completa, ou seja, os indivíduos pareados são completamente diferentes). Na hipótese nula de disposição espacial completamente aleatória (autocorrelação espacial ausente), $I(\mathrm{k})$ é assintoticamente gaussiano, com um valor esperado próximo a zero $\left[I_{\mathrm{e}}=-1 /(\mathrm{n}-1)\right]$, com $\mathrm{k}$ correspondendo às classes. Testes de significância foram feitos através do desvio padrão (Sokal \& Oden 1978a), usando o teste $\mathrm{Z}: \mathrm{Z}=$ $\left[I(\mathrm{k})-I_{\mathrm{e}}\right] / \mathrm{DP}$, com DP sendo o desvio padrão e $\mathrm{Z} \geq 1,98$ e $\mathrm{Z} \geq 2,56$, significativos a $5 \%$ e $1 \%$, respectivamente (Tokunaga \& Ohnishi 1992). Cabe ressaltar que o coeficiente I não é um parâmetro populacional e sim uma estatística de amostragem (Heywood 1991).

As comparações entre pares de indivíduos podem ser realizadas através de diferentes tipos de ponderação no cálculo do coeficiente $I$. Uma ponderação é escolhida para representar uma hipótese particular de estruturação espacial quando comparada com a hipótese nula de não haver estrutura. Nesse trabalho, foram utilizadas as comparações de vizinho mais próximo, conexão de Gabriel e todas as comparações dentro de uma classe de distância especificada.

$\mathrm{Na}$ análise do vizinho mais próximo, é dado o peso $\mathrm{w}=1$ a pares de indivíduos adjacentes e peso 0 a todos os demais pares possíveis (Sokal \& Oden 1978a). Segundo Dewey \& Heywood (1988), geralmente esse é o teste mais rigoroso para se detectar a presença de estrutura espacial. Sob isolamento pela distância, os vizinhos mais próximos devem prover as maiores autocorrelações, uma vez que o parentesco é esperado que diminua monotonicamente com a distância (Heywood 1991).

Já na conexão de Gabriel (Gabriel \& Sokal 1969), dois indivíduos A e B são pareados (recebem peso 1) se nenhum outro indivíduo ocorrer sobre, ou dentro do círculo cujo diâmetro é o segmento de reta AB (Sokal \& Oden 1978a). Nesse tipo de análise, mais indivíduos são pareados com indivíduos circundantes. No caso da conexão de Gabriel, a distância média entre indivíduos analisados é maior se comparada com a análise do vizinho mais próximo. Esse método é preferível ao do vizinho mais próximo quando a distância média entre localidades pareadas é pequena em relação à escala das variações espaciais a ser quantificada, ou seja, quando as localidades amostradas são suficientemente densas. Nesse caso, ambas as ponderações terão estimativas similares de autocorrelação, mas a conexão de Gabriel proverá uma estimativa mais precisa (Heywood 1991).

Na terceira forma de ponderação, utilizaram-se todas as comparações dentro de uma classe de distância especificada. Nessa análise, todas as comparações entre pares de indivíduos, cuja distância entre si apresentasse um valor incluído em um intervalo especificado, receberam peso 1, sendo que as demais comparações entre indivíduos receberam valor zero (0).

As classes de distância foram especificadas a fim de que cada uma apresentasse um número mínimo de conexões não inferior a 90. Desse modo, estabeleceram-se dez classes de distância entre indivíduos: 0-150 (1), 150-300 (2), 300-450 (3), 450-600 (4), 600-750(5), 750-900(6), 900-1050(7), 1050-1350 (8), 1350-1950 (9) e 1950-3500 (10) m.

As análises de autocorrelação espacial, através dos coeficientes $I$ de Moran, foram feitas utilizando o programa AUTOCORR versão 1.01 (1988) desenvolvido por J.S. Heywood (freeware, não publicado), e SAAP 4.3 (Wartenberg 1989). A significância do correlograma para cada loco, construído a partir de uma série de valores não independentes de $I$, foi determinada através do critério de Bonferroni (Sakai \& Oden 1983).

Adicionalmente, fez-se a análise de autocorrelação espacial multiloco e multialélica, baseada em métodos de distância genética, conforme apresentado por Smouse \& Peakall (1999) e Peakall et al. (2003), utilizando o programa GenAlEx v.5.1 (Peakall \& Smouse 2001) para os cálculos. Nessa análise, o coeficiente de autocorrelação " $r$ " foi utilizado, com sua respectiva significância sendo testada para cada alelo, em cada uma das mesmas classes de distância empregadas na análise anterior, através de intervalo de confiança de $95 \%$ gerado por 1000 permutações espaciais das 141 árvores amostradas. O intervalo de confiança de $95 \%$ para os coeficientes $r$ de cada classe foi gerado por 1.000 "bootstraps". A partir dessa abordagem, é possível determinar uma única medida de afinidade genética, incluindo-se todos os locos e todos os alelos na análise, com a obtenção do coeficiente $r$ total. Assim, permite-se testar se os genótipos de indivíduos sob estreita proximidade física são mais similares do que aqueles sob um maior distanciamento geográfico.

A abordagem multivariada foi empregada para fins comparativos porque, segundo Smouse \& Peakall (1999) e Peakall et al. (2003), essa apresentaria uma maior sensibilidade estatística do que os procedimentos clássicos de autocorrelação, reduzindo o ruído estocástico (variação entre locos e entre alelos) e fortalecendo o sinal espacial (padrão de afinidade genética). Dessa forma, o procedimento multialélico e multiloco mostraria o padrão essencial da estrutura genética espacial, extraindo toda a informação e suavizando os picos na curva gerada no correlograma. 


\section{Resultados}

Observa-se pelos dados apresentados na tabela 1 que, pelo método de vizinho mais próximo $\left(I_{\mathrm{vmp}}\right)$, houve 15 valores positivos de $I$ de Moran e nove negativos, enquanto que, pelo método de conexão de Gabriel $\left(I_{\mathrm{g}}\right)$, esses valores foram $14 \mathrm{e} 10$, respectivamente (tabela 1). Essa diferença encontrada entre os dois métodos foi promovida pelos diferentes sinais encontrados entre

Tabela 1. Coeficientes $I$ de Moran pelos dois métodos de pareamento de indivíduos: vizinho mais próximo $\left(I_{\mathrm{vmp}}\right)$ e conexão de Gabriel $\left(I_{\mathrm{g}}\right)$; distância média entre indivíduos pareados e número de conexões, em uma população natural de Cryptocarya moschata Nees do P.E. Carlos Botelho, S. Miguel Arcanjo, SP. ( $\mathrm{N}=141$ indivíduos $) . * \mathrm{p}<0,05$; $* * \mathrm{p}<0,01$.

Table 1. Moran's I coefficients from two methods for connecting the individuals: nearest-neighbor $\left(I_{\mathrm{vmp}}\right)$ and Gabriel-connected $\left(I_{\mathrm{g}}\right)$; average distance among pair-wise individuals and number of connections, in a natural population of Cryptocarya moschata Nees from P.E. Carlos Botelho, S. Miguel Arcanjo, SP. ( $N=141$ individuals). $* \mathrm{p}<0,05 ; * * \mathrm{p}<0,01$.

\begin{tabular}{|c|c|c|c|}
\hline Loco & Alelo & $I_{\mathrm{vmp}}$ & $I_{\mathrm{g}}$ \\
\hline Acp-3 & 1 & $0,287 * *$ & $0,240 * *$ \\
\hline Alp-1 & 1 & 0,104 & 0,094 \\
\hline Alp-3 & 1 & 0,129 & 0,104 \\
\hline Alp-4 & 1 & $0,691 * *$ & $0,412 * *$ \\
\hline Alp-5 & 1 & $-0,017$ & $-0,020$ \\
\hline Cat-1 & 1 & $-0,018$ & $-0,087$ \\
\hline Cat-2 & 1 & 0,095 & 0,081 \\
\hline Cat-3 & 1 & $-0,042$ & 0,052 \\
\hline Cat-4 & 1 & $-0,176$ & $-0,222 * *$ \\
\hline \multirow[t]{3}{*}{ Got-1 } & 1 & 0,046 & $-0,019$ \\
\hline & 2 & $-0,107$ & $-0,028$ \\
\hline & 3 & 0,109 & 0,010 \\
\hline \multirow[t]{3}{*}{ Got-2 } & 1 & $-0,041$ & $-0,072$ \\
\hline & 2 & $-0,034$ & 0,029 \\
\hline & 3 & 0,075 & 0,083 \\
\hline Po-1 & 1 & $0,211 *$ & $0,202 * *$ \\
\hline Po-2 & 1 & $0,205^{*}$ & $0,155^{*}$ \\
\hline Po-3 & 1 & $0,348 * *$ & $0,269 * *$ \\
\hline \multirow[t]{3}{*}{ Ро-4 } & 1 & $-0,089$ & $-0,038$ \\
\hline & 2 & 0,133 & 0,053 \\
\hline & 3 & 0,107 & $-0,015$ \\
\hline Po-5 & 1 & 0,046 & $-0,022$ \\
\hline Рpo-4 & 1 & $-0,113$ & $-0,001$ \\
\hline Ppo-5 & 1 & 0,048 & 0,042 \\
\hline \multicolumn{2}{|c|}{ Distância média (m) } & $37,30 \pm 15,80$ & $101,50 \pm 51,30$ \\
\hline \multicolumn{2}{|c|}{ Número de conexões } & 97 & 201 \\
\hline
\end{tabular}

os mesmos para os alelos de Cat-3, Got-1 alelo 1, Got-2 alelo 2, Po-4 alelo 3 e Po-5, uma vez que a distância média analisada para o método de vizinho mais próximo foi de $37,30 \pm 15,80$, enquanto que a do método de conexão de Gabriel foi de 101,50 \pm 51,30. Soma-se a isto, a existência dos mesmos cinco valores significativos para ambos os métodos (Acp-3, Alp-4, Po-1, Po-2 e Po-3), todos positivos.

A partir dos coeficientes $I$ de Moran para as dez classes de distância entre indivíduos, bem como os respectivos valores do critério de Bonferroni (tabela 2), fizeram-se os correlogramas de cada alelo. Dessa forma, na figura 2, apresentam-se os correlogramas obtidos para os locos Acp-3, Alp-1, Alp-3, Alp-4 e Alp-5. Na figura 3, são apresentados os correlogramas dos locos Cat-1, Cat-2, Cat-3 e Cat-4. Os locos Got-1 e Got-2, são apresentados na figura 4, assim como os locos Po-1, Po-2, Po-3 e Po-5 encontram-se na figura 5, e os locos Po-4, Ppo-4 e Ppo-5 na figura 6.

Dos 240 coeficientes $I$ de Moran obtidos da análise por classes de distância (24 freqüências em 10 classes), 77 foram significativos $(32,08 \%)$ ao nível de 5\% de probabilidade, sendo que, desses, 39 com valores positivos e 38 com valores negativos. Pelo critério de Bonferroni, 15 correlogramas mostraram-se significativos ao nível de 5\% de probabilidade (tabela 2). A primeira classe de distância apresentou valores positivos significativos para 11 alelos (Acp-3, Alp-3, Alp-4, Cat-1, Cat-2, Cat-3, Cat4, Po-1, Po-2, Po-3 e Po-4 alelo 3). As classes de distância 2 a 4,8 e 9 apresentaram preponderância de valores positivos significativos, enquanto as classes 6,7 e 10 apresentaram preponderância de valores negativos significativos.

Apenas quatro alelos não apresentaram valores significativos e nenhuma estruturação para todas as classes de distância analisadas (Alp-5, Got-2 alelo 1, Ppo-4 e Ppo-5), indicando flutuações aleatórias entre valores positivos e negativos, com nenhuma tendência clara de $I$ ser mais positivo para distâncias pequenas em oposição às grandes distâncias. Adicionalmente, os alelos de Alp-1, Got-1 alelo 3, Got-2 alelo 3, Po-2 e Po-4 alelo 1, apesar de apresentarem um ou dois valores significativos, seus correlogramas também mostraram-se sem estruturação espacial.

Por outro lado, os correlogramas dos alelos de Acp-3, Alp-3, Alp-4, Cat-1, Cat-2, Cat-3, Cat-4, Got-1 alelos 1 e 2, Got-2 alelo 2, Po-1, Po-3, Po-4 alelos 2 e 3 e Po-5 foram os que apresentaram autocorrelação espacial.

Pelo exame da tabela 3, dos coeficientes de correlação $r$ calculados a partir da análise multialélica e 
Tabela 2. Coeficientes $I$ de Moran para 10 classes de distância e para 24 alelos de 18 locos isoenzimáticos; distância média entre indivíduos pareados, desvio padrão e número de conexões, em uma população natural de Cryptocarya moschata Nees do P.E. Carlos Botelho, S. Miguel Arcanjo, SP. (N = 141 indivíduos). *p < 0,05; **p < 0,01; Classes de distância em metros: (1) 0-150; (2) 150-300; (3) 300-450; (4) 450-600; (5) 600-750; (6) 750-900; (7) 900-1050; (8) 1050-1350; (9) 1350-1950; (10) $1950-3500$.

Table 2. Moran's I coefficients for 10 classes of distance and for 24 alleles of 18 isozyme loci; average distance among pair-wise individuals, standard deviation and number of connections, in a natural population of Cryptocarya moschata Nees from P.E. Carlos Botelho, S. Miguel Arcanjo, SP. ( $\mathrm{N}=141$ individuals). *p < 0,05; **p < 0,01; ${ }^{\dagger}$ Distance classes in meters: (1) 0-150; (2) 150-300; (3) 300-450; (4) 450-600; (5) 600-750; (6) 750-900; (7) 900-1050; (8) 1050-1350; (9) 1350-1950; (10) 1950-3500.

\begin{tabular}{|c|c|c|c|c|c|c|c|c|c|c|c|}
\hline \multirow[b]{2}{*}{ Loco (alelo) } & \multicolumn{11}{|c|}{ Classes de distância ${ }^{\dagger}$} \\
\hline & 1 & 2 & 3 & 4 & 5 & 6 & 7 & 8 & 9 & 10 & Bonferroni \\
\hline Acp-3 & $0,14 * *$ & 0,02 & $-0,00$ & $-0,07 *$ & $-0,10^{* *}$ & $-0,02$ & $-0,07 *$ & $0,07 * *$ & $0,03 *$ & $-0,03 *$ & 0,001 \\
\hline Alp-1 & $-0,05$ & 0,03 & 0,01 & 0,01 & 0,01 & $-0,06$ & 0,03 & $-0,00$ & $-0,05^{*}$ & $-0,01$ & 0,371 \\
\hline Alp-3 & $0,22 * *$ & $-0,03$ & 0,01 & 0,00 & $0,06^{* *}$ & $-0,06$ & $-0,11 * *$ & 0,03 & $-0,02$ & $-0,06^{* *}$ & 0,000 \\
\hline Alp-4 & $0,37 * *$ & $0,09 * *$ & $0,12 * *$ & $0,05^{*}$ & 0,02 & $0,07 *$ & $0,08^{*}$ & $-0,11 * *$ & $-0,02$ & $-0,17 * *$ & 0,000 \\
\hline Alp-5 & $-0,00$ & $-0,02$ & $-0,01$ & 0,01 & 0,03 & $-0,06$ & $-0,03$ & 0,00 & $-0,01$ & $-0,01$ & 0,719 \\
\hline Cat-1 & $0,21 * *$ & 0,02 & $0,09 * *$ & $0,09 * *$ & $0,10^{* *}$ & $-0,17 * *$ & $-0,18 * *$ & $-0,03$ & $-0,11^{* *}$ & $-0,03^{*}$ & 0,000 \\
\hline Cat-2 & $0,28 * *$ & $-0,01$ & 0,03 & $0,06^{* *}$ & 0,03 & $-0,17 * *$ & $-0,08^{*}$ & $-0,02$ & $0,04^{*}$ & $-0,09 * *$ & 0,000 \\
\hline Cat-3 & $0,12 * *$ & $-0,02$ & $-0,04$ & $-0,07^{*}$ & 0,01 & $-0,08^{*}$ & $-0,01$ & $0,04 *$ & 0,01 & $-0,02$ & 0,010 \\
\hline Cat-4 & $0,11 * *$ & $0,17 * *$ & $-0,03$ & 0,01 & $-0,09 * *$ & $-0,23 * *$ & $-0,10 * *$ & 0,02 & $0,07 * *$ & $-0,02$ & 0,000 \\
\hline Got-1 (1) & 0,03 & 0,02 & $-0,02$ & 0,03 & $-0,03$ & $-0,01$ & $-0,16^{* *}$ & $0,04^{*}$ & $0,04^{*}$ & $-0,03^{*}$ & 0,000 \\
\hline Got-1 (2) & 0,03 & $-0,05$ & $-0,00$ & $-0,03$ & 0,00 & $-0,01$ & $-0,11 * *$ & $0,06^{* *}$ & $-0,01$ & $-0,01$ & 0,035 \\
\hline Got-1 (3) & $-0,04$ & 0,02 & $-0,04$ & $0,07 * *$ & $-0,07 *$ & $-0,02$ & 0,02 & $-0,01$ & 0,02 & $-0,01$ & 0,060 \\
\hline Got-2 (1) & $-0,05$ & $-0,04$ & 0,01 & $-0,03$ & 0,02 & $-0,01$ & 0,02 & $-0,03$ & $-0,00$ & 0,00 & 1,000 \\
\hline Got-2 (2) & 0,02 & 0,00 & $0,05^{*}$ & $-0,00$ & 0,01 & $-0,03$ & 0,01 & $-0,04$ & $-0,09 * *$ & $0,02 *$ & 0,002 \\
\hline Got-2 (3) & $-0,02$ & $-0,00$ & $-0,02$ & 0,02 & 0,02 & 0,00 & $-0,00$ & $-0,00$ & $-0,06^{*}$ & 0,00 & 0,105 \\
\hline Po-1 & $0,14 * *$ & $-0,05$ & $0,07 * *$ & $-0,04$ & $-0,04$ & 0,01 & 0,03 & $-0,03$ & $-0,01$ & $-0,02$ & 0,002 \\
\hline Po-2 & $0,10 * *$ & 0,01 & $-0,05$ & $-0,05$ & 0,03 & 0,03 & $-0,04$ & 0,03 & $-0,02$ & $-0,03^{*}$ & 0,068 \\
\hline Po-3 & $0,10 * *$ & $-0,01$ & $-0,05$ & $-0,08^{* *}$ & $0,07 * *$ & $-0,05$ & $-0,08^{*}$ & $-0,02$ & 0,02 & $-0,00$ & 0,048 \\
\hline Po-4 (1) & $-0,02$ & 0,03 & 0,03 & $-0,04$ & 0,03 & $-0,08^{*}$ & $-0,04$ & 0,01 & $-0,02$ & $-0,01$ & 0,360 \\
\hline Po-4 (2) & 0,05 & $0,07 *$ & 0,00 & $0,04 *$ & $-0,02$ & $-0,15^{* *}$ & $-0,12 * *$ & $-0,06^{*}$ & $0,06^{* *}$ & $-0,01$ & 0,002 \\
\hline Po-4 (3) & $0,09 * *$ & $0,09 * *$ & $-0,08^{*}$ & $-0,00$ & $-0,04$ & $-0,09 *$ & $-0,03$ & $-0,04$ & $0,03^{*}$ & 0,00 & 0,038 \\
\hline Po-5 & $-0,00$ & $-0,03$ & 0,04 & 0,03 & 0,01 & $-0,03$ & $-0,01$ & $-0,01$ & 0,02 & $-0,05 * *$ & 0,001 \\
\hline Ppo-4 & $-0,06$ & 0,01 & $-0,02$ & $-0,03$ & 0,01 & $-0,02$ & $-0,01$ & 0,00 & 0,01 & $-0,00$ & 1,000 \\
\hline Ppo-5 & 0,05 & 0,01 & $-0,04$ & $-0,04$ & $-0,02$ & $-0,02$ & $-0,05$ & 0,02 & $-0,00$ & 0,00 & 0,882 \\
\hline I Médio & 0,08 & 0,01 & 0,00 & $-0,00$ & 0,00 & $-0,05$ & $-0,04$ & $-0,00$ & $-0,00$ & $-0,02$ & \\
\hline I Máximo & 0,37 & 0,17 & 0,12 & 0,09 & 0,10 & 0,07 & 0,08 & 0,07 & 0,07 & 0,02 & \\
\hline I Mínimo & $-0,06$ & $-0,05$ & $-0,08$ & $-0,08$ & $-0,10$ & $-0,23$ & $-0,18$ & $-0,11$ & $-0,11$ & $-0,17$ & \\
\hline Distância média (m) & 73,40 & 235,80 & 376,70 & 514,20 & 676,20 & 822,60 & 972,60 & 1182,20 & 1609,20 & 2709,10 & \\
\hline Desvio padrão & 13,07 & 13,70 & 14,00 & 13,33 & 12,70 & 14,20 & 13,03 & 25,69 & 53,07 & 125,14 & \\
\hline Número de conexões & 522,00 & 605,00 & 846,00 & $891,00 \quad 1$ & 1019,00 & 552,00 & 549,00 & 1105,00 & 1304,00 & 2265,00 & \\
\hline
\end{tabular}

multilocos, observa-se que a maior parte dos valores significativos concordou com as estimativas dos coeficientes $I$ de Moran, apresentados na tabela 2 para as mesmas classes de distância. Apreende-se que os correlogramas gerados, a partir do coeficiente $r$ total e do $I$ de Moran médio para todos os alelos, foram bastante semelhantes. No entanto, para os coeficientes $r$ totais, as classes de distância 1, 6, 7 e 10 foram significativas (figura 7).

\section{Discussão}

Pelos dados apresentados na tabela 1, apesar de haver maior número de valores positivos de coeficientes $I$ de Moran (localidades adjacentes são similares) do que o de negativos (diferenças marcantes entre pares adjacentes) e por serem apenas cinco alelos com valores significativos dentre os 24 analisados, a estruturação espacial mostrou-se aleatória. 


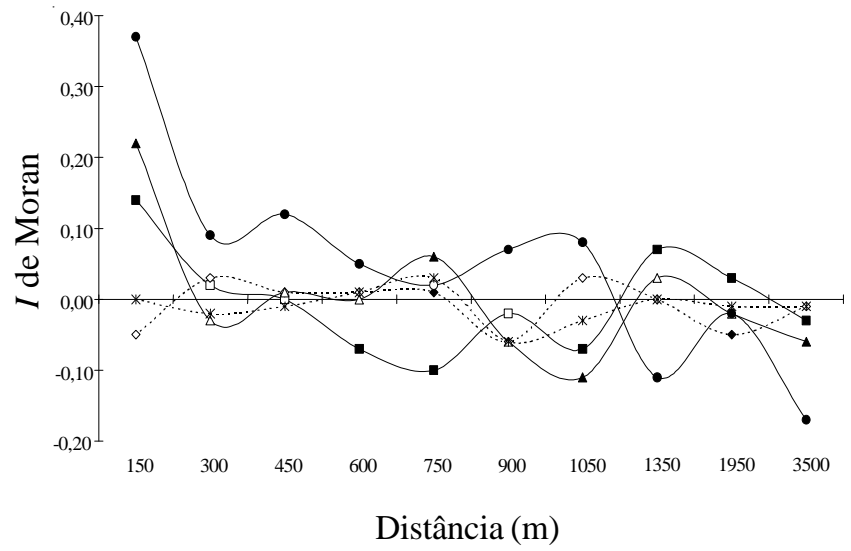

Figura 2. Correlogramas espaciais dos alelos de Acp-3, Alp-1, Alp-3, Alp-4 e Alp-5 para 10 classes de distância em uma população natural de Cryptocarya moschata Nees do PECB. Polígonos e círculos vazios indicam autocorrelação não significativa. Alinha tracejada indica ausência de significância do correlograma, segundo o critério de Bonferroni. - - Acp-3, - - - Alp-1, - $\mathbf{\Delta - A l p - 3 , - \bullet - A l p - 5 . ~}$

Figure 2. Spatial correlograms of alleles from Acp-3, Alp-1, Alp-3, Alp-4 and Alp-5, for 10 distance classes in a natural population of Cryptocarya moschata Nees at PECB. Empty polygons and circles indicate non significant autocorrelation. Dashed line indicates non significant correlogram according to Bonferroni's criterion. - - Acp-3, - - - Alp-1,-A-Alp-3, $-\bullet-$ Alp-5.

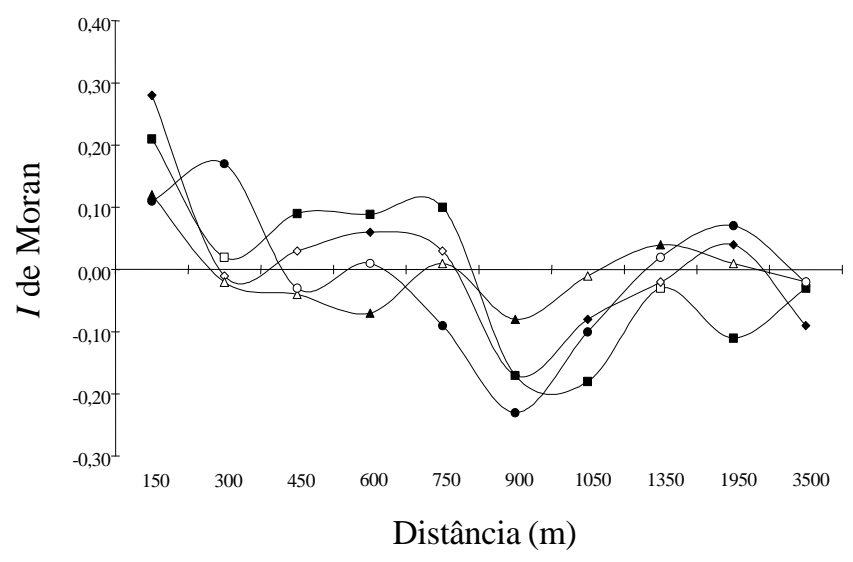

Figura 3. Correlogramas espaciais dos alelos de Cat-1, Cat2, Cat-3 e Cat-4, para 10 classes de distância em uma população natural de Cryptocarya moschata Nees do PECB. Polígonos e círculos vazios indicam autocorrelação não

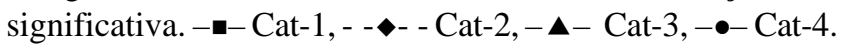

Figure 3. Spatial correlograms of alleles from Cat-1, Cat-2, Cat-3 and Cat-4, for 10 distance classes, in a natural population of Cryptocarya moschata Nees at PECB. Empty polygons and circles indicate non significant autocorrelation.

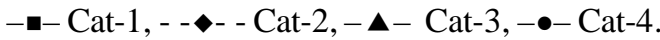

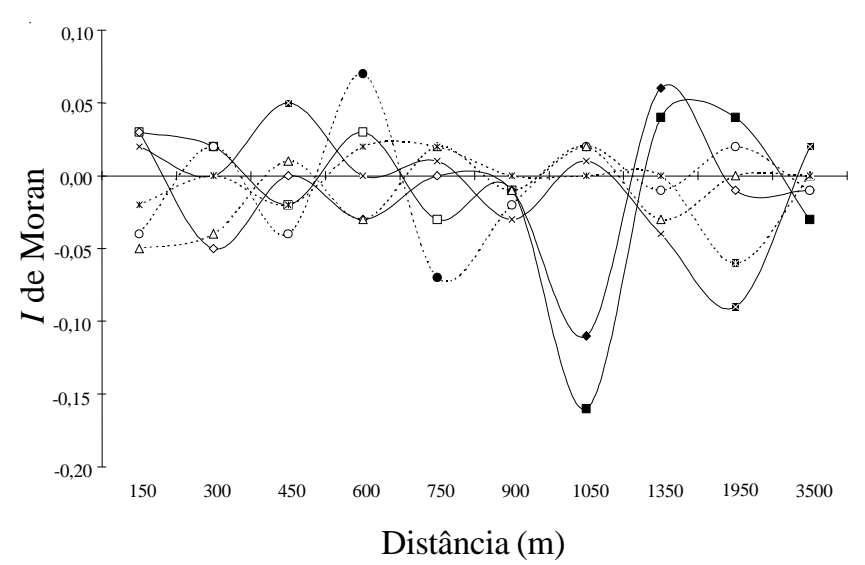

Figura 4. Correlogramas espaciais dos alelos 1, 2 e 3 de Got1 e de Got-2, para 10 classes de distância, em uma população natural de Cryptocarya moschata Nees do PECB. Polígonos e círculos vazios indicam autocorrelação não significativa. A linha tracejada indica ausência de significância do correlograma, segundo o critério de Bonferroni. - - Got-1(1), - - - - Got-1 (2), - - •- - Got-1 (3), - - $\mathbf{-}$ - - Got-2 (1), - 囚-Got-2 (2), - - - - Got-2 (3).

Figure 4. Spatial correlograms of alleles 1, 2 and 3 from Got-1 and Got-2, for 10 distance classes, in a natural population of Cryptocarya moschata Nees at PECB. Empty polygons and circles indicate non significant autocorrelation. Dashed line indicates non significant correlogram according to Bonferroni's criterion. - - - Got-1(1), - - - - Got-1 (2), - - •- -

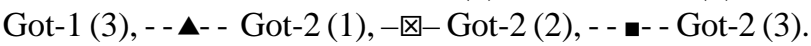

A partir dos coeficientes $I$ obtidos para as classes de distância (tabela 2), tem-se que a maioria dos alelos não apresentou estruturação espacial nas classes de menores distâncias (1 a 3). Conforme Dewey \& Heywood (1988), uma possível explicação para esse resultado, que contradiz a predição de que as populações de plantas estão subdivididas em demes locais ou vizinhanças de indivíduos aparentados (Levin \& Kerster 1974), é que a diferenciação local estaria sendo evitada por algum tipo de seleção estabilizadora operante em uma pequena escala espacial. Nesse sentido, a vantagem de heterozigotos, que tem sido uma das propostas para a explicação da homogeneidade espacial em grandes escalas (Bulmer 1973), teria o mesmo efeito em escala local. Na literatura, há várias evidências de vantagem de heterozigotos de locos isoenzimáticos (Bush et al. 1987). Dessa forma, essa explicação aplicar-se-ia apenas ao alelo de Ppo-4, o único com excesso significativo de heterozigotos em relação às proporções de Equilíbrio de Hardy-Weinberg (Moraes et al. 2002) e que não apresentou estrutura espacial. No entanto, seria necessário, também, vantagem seletiva do 


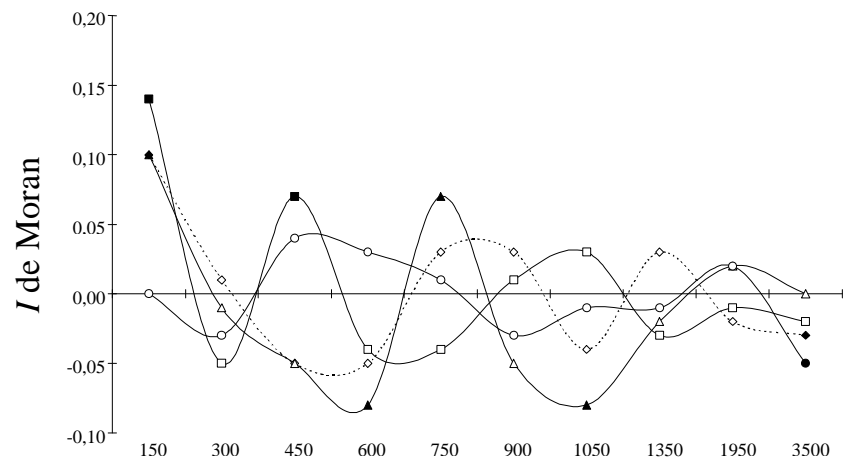

Distância (m)

Figura 5. Correlogramas espaciais dos alelos de Po-1, Po-2, Po-3 e Po-5 para 10 classes de distância em uma população natural de Cryptocarya moschata Nees do PECB. Polígonos e círculos vazios indicam autocorrelação não significativa. A linha tracejada indica ausência de significância do correlograma, segundo o critério de Bonferroni. -- - Po-1,

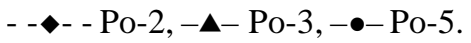

Figure 5. Spatial correlograms of alleles from Po-1, Po-2, Po-3 and Po-5 for 10 distance classes in a natural population of Cryptocarya moschata Nees at PECB. Empty polygons and circles indicate non significant autocorrelation. Dashed line indicates non significant correlogram according to Bonferroni's criterion. - - - Po-1, - - - - Po-2, - $\mathbf{-}$ - Po-3, $-\bullet-$ Po-5.

homozigoto 22 em relação ao 11 , levando-se em consideração as desigualdades nas freqüências alélicas, o que não se confirmou (Moraes et al. 2002, P.L.R. Moraes, dados não publicados). Com isso, o excesso significativo de heterozigotos do loco Ppo-4 não estaria associado à vantagem seletiva destes e sim a uma possível seleção contra os homozigotos 22 .

Uma outra explicação possível seria a de que a seleção natural, atuando em escala local, favoreceria os genótipos mais adaptados a determinados microhábitats, estruturando conseqüentemente a população. Epperson \& Allard (1989) encontraram evidências desse fato para alguns alelos em Pinus contorta Dougl. ex Loud. ssp. latifolia (S. Wats.) E. Murray, apesar de a distribuição de genótipos ter sido em geral aleatória. No entanto, para o presente estudo, não há indicações de que isso esteja ocorrendo. Adicionalmente, para se poder afirmar com segurança que a estrutura espacial encontrada deve-se ao efeito de seleção local, várias gerações deveriam ser estudadas (Tokunaga \& Ohnishi 1992). Com a possível variação do padrão nas gerações seguintes, a hipótese de seleção para microhábitats específicos, como

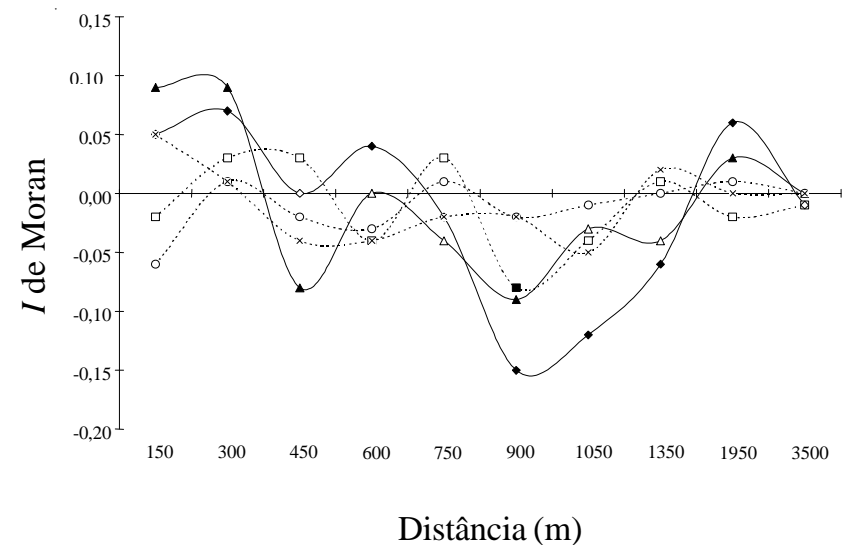

Figura 6. Correlogramas espaciais dos alelos 1, 2 e 3 de Po-4 e de Ppo-4 e Ppo-5 para 10 classes de distância em uma população natural de Cryptocarya moschata Nees do PECB. Polígonos e círculos vazios indicam autocorrelação não significativa. A linha tracejada indica ausência de significância do correlograma, segundo o critério de Bonferroni. - -₫- - Po-4 (1), - - Po-4 (2),

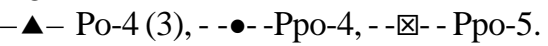

Figure 6. Spatial correlograms of alleles 1, 2 and 3 from Po-4, and from Ppo-4 and Ppo-5, for 10 distance classes, in a natural population of Cryptocarya moschata Nees at PECB. Empty polygons and circles indicate non significant autocorrelation. Dashed line indicates non significant correlogram according to Bonferroni's criterion. - - - - Po-4 (1), - - - Po-4 (2), - $\mathbf{\Delta -}$ Po-4 (3), - -॰- - Ppo-4, - - $\-$ - Ppo-5.

geradora da agregação de alelos, poderia ser descartada.

Ainda, uma outra explicação para essa ausência de estrutura espacial para as classes de menores distâncias seria a do fluxo gênico estar ocorrendo em quantidade suficiente para evitar a divergência aleatória das frequiências gênicas locais para esses locos (Dewey \& Heywood 1988, Epperson \& Allard 1989, Doligez \& Joly 1997).

As diferenças encontradas entre os correlogramas são esperadas, uma vez que os diferentes alelos e os diferentes locos provavelmente estão sujeitos a diferentes forças evolutivas (Slatkin \& Arter 1991). Como apontado por Sokal (1979), a autocorrelação positiva a pequenas distâncias, em que localidades vizinhas assumem usualmente valores similares, pode surgir não apenas devido a gradientes ("clines"), mas também a partir de localidades cujos diâmetros excedam a distância entre os pontos, porém são menores do que quaisquer vazios entre as localidades. Com isto, o único alelo que se mostrou com nítido padrão de distribuição espacial em gradiente foi o de Alp-4.

De maneira diversa, a autocorrelação espacial negativa a pequenas distâncias, envolvendo usualmente 


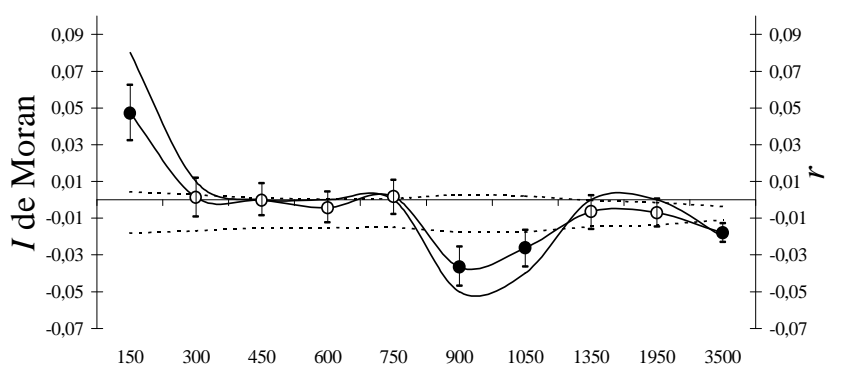

Distância (m)

Figura 7. Correlograma da média de 24 alelos analisados pelo $I$ de Moran e correlograma total dos coeficientes $r$, a partir da análise multivariada de todos os locos e de todos os alelos, para 10 classes de distância, em uma população natural de Cryptocarya moschata Nees do PECB. Círculos vazios indicam autocorrelação não significativa. As linhas tracejadas indicam o intervalo de confiança para os coeficientes $r$ ao nível de $95 \%$. Barras de erro de $95 \%$ de confiança sobre $r$ obtidas de 1000 "bootstraps". - I, -•-r, ------ I.C. 95\%.

Figure 7. Average correlogram for 24 alleles analysed from Moran's I coefficient, and total correlogram of $r$ coefficient from multivariate analysis for all loci and all alleles, for 10 distance classes, in a natural population of Cryptocarya moschata Nees at PECB. Empty circles indicate non significant autocorrelation. Dashed lines indicate the confidence interval for $r$ coefficient at $95 \%$ level. $95 \%$ confidence error bars about $r$ as determined by 1000 bootstraps. - I, - $\bullet-r$, ------ I.C. $95 \%$.

vizinhos diferentes, sugere uma mistura de localidades heterogêneas menores em diâmetro médio do que a distância entre as localidades (Sokal 1979) ou, dito de outra forma, com um intervalo de amostragem muito grande para se detectar associações (Legendre \& Fortin 1989). Esse tipo de situação não ocorreu no presente trabalho, apesar de os alelos dos locos Alp-1, Alp-3, Alp-5, Cat-2, Cat-3, Got-1 alelos 2 e 3, Got-2 alelos 1 e 3, Po-1, Po-3, Po-4 alelo 1, Po-5 e Ppo-4 apresentarem valores negativos para as duas menores classes de distância, porém não significativos.

A autocorrelação espacial positiva para grandes distâncias tende a ser o resultado de um arranjo regular de localidades de maneira que os valores são aproximadamente repetidos, como cada uma das encostas de uma montanha ou de um vale (Sokal 1979, Legendre \& Fortin 1989) ou, dito de outra forma, se o gradiente for circular ou simétrico (Sokal \& Oden 1978b). Essa situação pode estar associada aos alelos de Acp-3, Alp-4, Cat-3, Cat-4, Got-1 alelos 1 e 2, Got-2 alelo 2 e Po-4 alelos 2 e 3 .

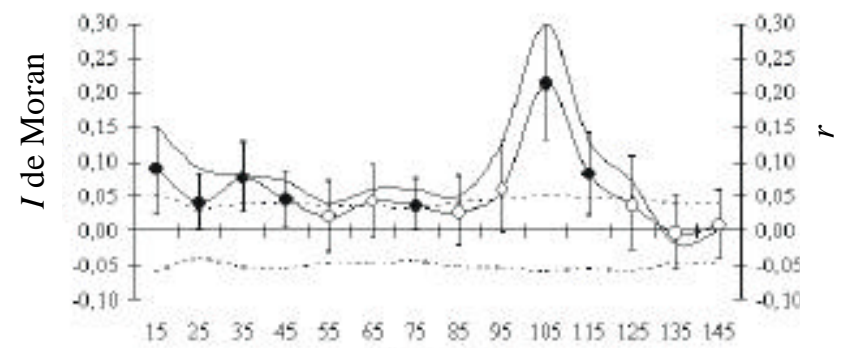

Distância (m)

Figura 8: Correlograma da média de 24 alelos analisados pelo $I$ de Moran e correlograma total dos coeficientes $r$, a partir da análise multivariada de todos os locos e todos os alelos, para 14 classes de distância, em uma população natural de Cryptocarya moschata Nees do PECB. Círculos vazios indicam autocorrelação não significativa. As linhas tracejadas indicam o intervalo de confiança para os coeficientes $r$ ao nível de $95 \%$. Barras de erro de $95 \%$ de confiança sobre $r$ obtidas de 1000 "bootstraps". - I, -•-r, ------ I.C. 95\%.

Figure 8. Average correlogram for 24 alleles analysed from Moran's $I$ coefficient, and total correlogram of $r$ coefficient from multivariate analysis for all loci and all alleles, for 14 distance classes, in a natural population of Cryptocarya moschata Nees at PECB. Empty circles indicate non significant autocorrelation. Dashed lines indicate the confidence interval for $r$ coefficient at $95 \%$ level. 95\% confidence error bars about $r$ as determined by 1000 bootstraps. - I, - $\bullet-r$, ------ I.C. $95 \%$.

Por sua vez, a autocorrelação espacial negativa para grandes distâncias, excetuada de um contexto de "cline", também pode ocorrer a partir da influência de certas localidades ocorrendo próximas das bordas da área amostrada (Epperson \& Clegg 1986, Perry \& Knowles 1991). Dessa forma, essa poderia ser uma explicação para o observado para os alelos de Acp-3, Alp-1, Alp-3, Cat-1, Cat-2, Got-1 alelo 1, Got-2 alelo 3, Po-2 e Po-5.

Por outro lado, o alelo de Alp-4, como apontado anteriormente, poderia estar refletindo a existência de gradiente, com as localidades mais dissimilares encontrando-se mais afastadas entre si. Contudo, Legendre et al. (1990) fazem distinção entre o que denominaram de gradientes "falsos" e "verdadeiros". No presente estudo, assumindo-se que a estrutura espacial em forma de gradiente, para o alelo de Alp-4, seria o resultado da dinâmica populacional (modelo de controle biótico, usualmente empregado em estudos de escalas pequenas; Southwood 1987), em contraposição ao modelo de controle ambiental, em que fatores 
Tabela 3. Coeficientes de correlação $r$ calculados para 10 classes de distância e para 18 locos isoenzimáticos em uma população natural de Cryptocarya moschata Nees do P.E. Carlos Botelho, S. Miguel Arcanjo, SP. (N = 141 indivíduos). ${ }^{*} \mathrm{p}<0,05 ;{ }^{\dagger}$ Classes de distância em metros: (1) 0-150; (2) 150-300; (3) 300-450; (4) 450-600; (5) 600-750; (6) 750-900; (7) 900-1050; (8) 1050-1350; (9) 1350-1950; (10) 1950-3500.

Table 3. Correlation coefficients $r$ calculated for 10 classes of distance and for 18 isozyme loci in a natural population of Cryptocarya moschata Nees from P.E. Carlos Botelho, S. Miguel Arcanjo, SP. (N = 141 individuals). * $\mathrm{p}<0,05 ;{ }^{\dagger}$ Distance classes in meters: (1) 0-150; (2) 150-300; (3) 300-450; (4) 450-600; (5) 600-750; (6) 750-900; (7) 900-1050; (8) 1050-1350; (9) 1350-1950; (10) 1950-3500.

\begin{tabular}{|c|c|c|c|c|c|c|c|c|c|c|}
\hline \multirow[b]{2}{*}{ Loco } & \multicolumn{10}{|c|}{ Classes de distância ${ }^{\dagger}$} \\
\hline & 1 & 2 & 3 & 4 & 5 & 6 & 7 & 8 & 9 & 10 \\
\hline Acp-3 & $0,118^{*}$ & 0,020 & $-0,004$ & $-0,083^{*}$ & $-0,083^{*}$ & $-0,014$ & $-0,067$ & $0,053 *$ & 0,036 & $-0,040$ \\
\hline Alp-1 & $-0,045$ & 0,012 & 0,002 & 0,011 & 0,005 & $-0,034$ & 0,018 & $-0,010$ & $-0,036$ & 0,009 \\
\hline Alp-3 & $0,229 *$ & 0,012 & 0,010 & $-0,010$ & $0,049 *$ & $-0,072$ & $-0,087 *$ & 0,010 & $-0,048$ & $-0,076^{*}$ \\
\hline Alp-4 & $0,459 *$ & $0,094^{*}$ & $0,138^{*}$ & $0,065^{*}$ & 0,030 & $0,098 *$ & $0,164 *$ & $-0,138^{*}$ & $-0,033$ & $-0,080 *$ \\
\hline Alp-5 & $-0,004$ & $-0,034$ & $-0,011$ & 0,013 & 0,030 & $-0,047$ & $-0,034$ & 0,002 & $-0,016$ & 0,017 \\
\hline Cat-1 & $0,165^{*}$ & 0,009 & $0,056^{*}$ & $0,072 *$ & $0,078^{*}$ & $-0,079 *$ & $-0,109 *$ & $-0,022$ & $-0,074 *$ & $-0,054^{*}$ \\
\hline Cat-2 & $0,249 *$ & $0,062 *$ & 0,016 & 0,025 & $-0,014$ & $-0,134 *$ & $-0,079 *$ & $-0,014$ & 0,010 & $-0,060 *$ \\
\hline Cat-3 & $0,106^{*}$ & $-0,012$ & $-0,032$ & $-0,048$ & 0,008 & $-0,064$ & $-0,009$ & 0,019 & $-0,004$ & $-0,016$ \\
\hline Cat-4 & $0,104 *$ & $0,110^{*}$ & $-0,015$ & 0,005 & $-0,061^{*}$ & $-0,162 *$ & $-0,092 *$ & 0,010 & $0,059 *$ & $-0,038^{*}$ \\
\hline Got-1 & 0,001 & $-0,002$ & $-0,013$ & 0,007 & $-0,039$ & $-0,033$ & $-0,040$ & 0,023 & 0,019 & $-0,020$ \\
\hline Got-2 & $-0,014$ & $-0,015$ & 0,003 & 0,001 & 0,014 & $-0,005$ & 0,008 & $-0,015$ & $-0,039 *$ & 0,004 \\
\hline Po-1 & $0,127 *$ & $-0,051$ & $0,063^{*}$ & $-0,049$ & $-0,029$ & $-0,012$ & 0,032 & $-0,027$ & $-0,007$ & $-0,024$ \\
\hline Po-2 & $0,097 *$ & $-0,009$ & $-0,045$ & $-0,043$ & $0,059 *$ & 0,050 & $-0,049$ & 0,025 & $-0,030$ & $-0,033$ \\
\hline Po-3 & $0,145^{*}$ & $-0,005$ & $-0,010$ & $-0,033$ & $0,053^{*}$ & $-0,019$ & $-0,086^{*}$ & $-0,069 *$ & $-0,029$ & $0,018^{*}$ \\
\hline Po-4 & 0,024 & $0,056^{*}$ & $-0,016$ & 0,004 & $-0,011$ & $-0,087^{*}$ & $-0,041$ & $-0,027$ & 0,018 & $-0,011$ \\
\hline Po-5 & 0,004 & $-0,052$ & 0,028 & 0,014 & 0,019 & $-0,014$ & 0,008 & $-0,024$ & 0,019 & $-0,036^{*}$ \\
\hline Ppo-4 & $-0,055$ & 0,005 & $-0,025$ & $-0,031$ & 0,013 & $-0,024$ & $-0,010$ & 0,000 & 0,007 & $-0,002$ \\
\hline Ppo-5 & 0,021 & 0,016 & $-0,029$ & $-0,030$ & $-0,029$ & $-0,016$ & $-0,051$ & 0,021 & 0,003 & 0,001 \\
\hline Total & $0,047 *$ & 0,001 & 0,000 & $-0,004$ & 0,002 & $-0,037 *$ & $-0,026^{*}$ & $-0,007$ & $-0,007$ & $-0,018^{*}$ \\
\hline Número de conexões & 522 & 605 & 846 & 891 & 1019 & 551 & 550 & 1105 & 1304 & 2265 \\
\hline
\end{tabular}

ambientais seriam os promotores da estruturação espacial, usualmente empregado em estudos de grande escala, então a estrutura encontrada é melhor considerada como sendo a de um falso gradiente (autocorrelação).

O ponto em que o correlograma cruza a abscissa (desvio padrão $=0$ ), ou troca de sinal, é considerado uma estimativa operacional do comprimento do menor lado de um polígono irregular que representa uma área média amostrada. Dessa forma, pela investigação dos correlogramas obtidos, observa-se que grande parte dos alelos enquadrou-se entre as classes de distância 1 a 3 (18 alelos), com distâncias médias de 73,4 m, 235,8 m e $376,7 \mathrm{~m}$ respectivamente, tendo uma preponderância de mudanças de sinal + para o $-(16 \times 8)$. Ainda, dos 13 alelos que apresentaram autocorrelações significativas precedentes à mudança de sinal, todos são + , indicando mais uma vez uma tendência dos indivíduos mais próximos serem mais similares geneticamente entre si, em relação aos mais distantes, o que sugere um possível parentesco entre os mesmos. Os alelos discrepantes, em relação a essa tendência observada para os demais, foram os de Alp-4, Alp-5, Cat-1, Got-2 alelos 2 e 3 e Po-4 alelo 2, com as mudanças de sinal ocorrendo nas classes 7/8, 3/4, 5/6, 3/4, 3/4 e 4/5, respectivamente. No entanto, a partir do correlograma obtido através do $I$ concensual dos 24 alelos analisados (figura 7), tem-se que o cruzamento da abscissa ocorreu próximo da classe de distância $3(450 \mathrm{~m})$. Pelo correlograma dos coeficientes $r$ totais, o cruzamento da abscissa ocorreu em torno dos 300 m. Essa distância seria uma indicação do distanciamento mínimo entre os indivíduos a serem coletados, visando-se amostragem para obtenção de variabilidade genética. Porém, observa-se na figura 7 que a partir de $750 \mathrm{~m}$ houve uma acentuada queda na curva do correlograma, culminando na maior 
autocorrelação negativa em torno de $900 \mathrm{~m}$. Com isto, para a população em questão, uma amostragem de indivíduos distanciados a partir de $750 \mathrm{~m}$ entre si promoveria uma maior probabilidade de aquisição de sementes com maior variabilidade genética.

Como indicado por Endler (1977), dentre outros, a distribuição espacial da variabilidade genética dentro de uma população pode influenciar significativamente a dinâmica evolutiva da mesma. Todavia, Slatkin \& Arter (1991) relacionaram uma série de limitações para o uso das análises "clássicas" de autocorrelação espacial em estudos de genética de população. Os problemas principais, segundo esses autores, são que os processos genéticos populacionais são inerentemente estocásticos, as freqüencias alélicas para os diferentes locos são afetadas por diferentes processos e os modelos não têm levado em consideração todas as fontes de variação presentes nos correlogramas. De qualquer forma, esse método poderia ser útil para uma análise exploratória dos dados, ao invés de ser utilizado para se tirar conclusões acerca dos padrões detectados.

Conforme discutido por Smouse \& Peakall (1999), a estrutura espacial determinada pelas abordagens clássicas raramente se mostra coerente entre os diferentes locos, sendo geralmente fraca, o que seria mais um reflexo da falta de sensibilidade dos procedimentos estatísticos empregados para a detecção da mesma, podendo ser outra provável explicação para a ausência de estrutura espacial encontrada no presente estudo. Porém, com o novo procedimento desenvolvido por esses autores, tratando o conjunto dos dados genéticos como um todo, em um método multivariado genérico para a análise de autocorrelação espacial, haveria uma redução do ruído estocástico existente (alelo-a-alelo e loco-a-loco), permitindo a obtenção de média global dos vários sistemas estocasticamente variáveis. Através desse, foi possível testar se os genótipos dos indivíduos em estreita proximidade física são mais similares do que aqueles sob um maior distanciamento espacial.

Como apontado anteriormente, os resultados obtidos pelas diferentes abordagens mostraram-se qualitativamente similares (correlogramas com curvas semelhantes; figuras 7,8), uma vez que o coeficiente de correlação $r$ está estreitamente relacionado ao coeficiente $I$ de Moran (Smouse \& Peakall 1999), sendo que o primeiro, no entanto, é completamente geral (multivariado). Observa-se na figura 7, contudo, que o correlograma gerado através da metodologia de análise multivariada apresentou estrutura espacial significativa para a primeira classe de distância (10 locos significativos: Acp-3, Alp-3, Alp-4, Cat-1, Cat-2, Cat-3, Cat-4, Po-1, Po-2 e Po-3), que compreende os indivíduos amostrados e distanciados até $150 \mathrm{~m}$. Como para essa classe de distância houve 522 conexões e muitos dos indivíduos amostrados encontram-se separados a distâncias de algumas dezenas de metros $(\mathrm{N}=178$; $2,64 \mathrm{~m} \leq x \geq 50 \mathrm{~m}$ ), investigou-se a autocorrelação espacial através dos $I$ de Moran e dos coeficientes $r$ para 14 classes de distância de $10 \mathrm{~m}$, compreendidas dentro desse limite de $150 \mathrm{~m}$, de maneira que a primeira classe incluiu todas as distâncias do intervalo 0-15 m, seguida pelo intervalo $15-25 \mathrm{~m}$ e assim por diante até o intervalo 135-145 m. Novamente, houve concordância de valores significativos para a maioria dos coeficientes obtidos pelas duas abordagens, sendo que na figura 8 percebe-se a semelhança qualitativa entre os correlogramas dos $I$ de Moran médios e dos coeficientes $r$ totais. Através dos coeficientes $r$ denota-se também que houve autocorrelação espacial positiva na maioria das classes, incluindo as de menores distâncias, indicando que os agrupamentos de árvores de $C$. moschata, que se encontram separadas a distâncias menores que $50 \mathrm{~m}$, são constituídos por indivíduos semelhantes geneticamente e provavelmente aparentados.

Essa similaridade genética entre os indivíduos próximos geograficamente pode ser explicada pela polinização que em Cryptocarya moschata, é feita provável e predominantemente por pequenos insetos, como moscas e pequenas abelhas, o que promoveria fluxo de pólen a curtas distâncias (Moraes et al. 1999). Adicionalmente, a espécie apresenta flores hermafroditas, autocompatíveis, com sistema de cruzamento misto, predominantemente alogâmico (taxa de cruzamento multilocos $\hat{t}_{\mathrm{m}}=0,884 \pm 0,034$ ), com endogamia biparental em torno de $16 \%$, para a população analisada do PECB (Moraes \& Monteiro 2002). A existência de estrutura espacial, na presença de fluxo de pólen homogeneizador, pode ser conseqüência de dispersão restrita de sementes (Smouse \& Peakall 1999), o que se aplicaria à C. moschata, pelo fato de a dispersão de diásporos ocorrer preponderantemente por gravidade sob as árvores-mãe, o que poderia promover um recrutamento de indivíduos aparentados a curtas distâncias.

No entanto, Moraes et al. (2002) indicaram também que a dispersão de sementes de Cryptocarya moschata promovida por Brachyteles arachnoides (Primates Cebidae) pode proporcionar tanto uma alta heterogeneidade genética entre locais de recrutamento de novos indivíduos, quanto uma menor heterogeneidade genética relativa à anterior, que estariam associadas aos 
diferentes comportamentos: 1) de alimentação de frutos de uma única árvore, com subseqüente deposição de vários diásporos (ao menos meios-irmãos) em locais que poderiam ser adequados para um novo recrutamento; ou 2) de alimentação de frutos de várias árvores, com deposição de diásporos ao longo da área de uso, respectivamente. Dessa forma, uma combinação dos diferentes comportamentos alimentares desses animais ao longo da população amostrada poderia estar contribuindo para a estruturação espacial da população, principalmente a pequenas distâncias. Ainda, poderia explicar também o alto valor de $r$ encontrado em torno de $105 \mathrm{~m}$ (figura 8), indicando uma maior similaridade genética entre os indivíduos. Esse distanciamento poderia estar associado aos locais de maior probabilidade de recrutamento de novos indivíduos, de acordo com o modelo de escape de Janzen-Connel (locais de defecação distanciados das árvores-mãe; Moraes \& Paoli 1995, Moraes \& Paoli 1999), conforme discutido por Moraes et al. (2002), a partir da análise de indivíduos juvenis oriundos de diásporos dispersados por Brachyteles.

Pelo exposto, denota-se que os resultados obtidos através do método multivariado concordaram com os argumentos apresentados por Smouse \& Peakall (1999) e Peakall et al. (2003), demonstrando uma melhor adequação para a investigação da similaridade dos genótipos em função do distanciamento geográfico entre os indivíduos.

Conclui-se que a população analisada de C. moschata apresentou estrutura genética espacial a pequenas distâncias de até $150 \mathrm{~m}$ entre os indivíduos, indicando que os mesmos devem ser aparentados. Frente a isto, para uma amostragem visando-se aquisição de diversidade genética, a coleta de indivíduos distanciados além de $750 \mathrm{~m}$ entre si seria recomendável, por ser a classe de distância a partir da qual os indivíduos se mostraram mais diferenciados geneticamente.

Agradecimentos - Ao Instituto Florestal de São Paulo, pela autorização de coleta no Parque Estadual Carlos Botelho. À Capes, pela bolsa de doutorado a P.L.R. de Moraes.

\section{Referências bibliográficas}

BULMER, M.G. 1973. Geographical uniformity of protein polymorphisms. Nature 241:199-200.

BUSH, R.M., SMOUSE, P.E. \& LEDIG, F.T. 1987. The fitness consequences of multiple-locus heterozygosity: the relationship between heterozygosity and growth rate in pitch pine (Pinus rigida Mill.). Evolution 41:787-798.
CAMPBELL, D.R. \& DOOLEY, J.L. 1992. The spatial scale of genetic differentiation in a hummingbird-pollinated plant: comparison with models of isolation by distance. The American Naturalist 139:735-748.

CLIFF, A.D. \& ORD, J.K. 1973. Spatial autocorrelation. Pion, London.

DEWEY, S.E. \& HEYWOOD, J.S. 1988. Spatial genetic structure in a population of Psychotria nervosa. I. Distribution of genotypes. Evolution 42:834-838.

DOLIGEZ, A. \& JOLY, H.I. 1997. Genetic diversity and spatial structure within a natural stand of a tropical forest tree species, Carpa procera (Meliaceae), in French Guiana. Heredity 79:72-82.

EASTMAN, J.R. 1992. IDRISI version 4.1. Technical reference manual. Graduate School of Geography, Clark University, Worcester.

ENDLER, J.A. 1977. Geographic variation, speciation, and clines. Princeton University Press, Princeton.

EPPERSON, B.K. 1990. Spatial autocorrelation of genotypes under directional selection. Genetics 124:757-771.

EPPERSON, B.K. 1995. Fine-scale spatial structure correlations for individual genotypes differ from those for local gene frequencies. Evolution 49:1022-1026.

EPPERSON, B.K. \& ALLARD, R.W. 1989. Spatial autocorrelation analysis of the distribution of genotypes within populations of lodgepole pine. Genetics 121:369-377.

EPPERSON, B.K. \& CLEGG, M.T. 1986. Spatial autocorrelation analysis of flower color polymorphisms within substructured populations of morning glory (Ipomoea purpurea). The American Naturalist 128:840-858.

GABRIEL, K. \& SOKAL, R.R. 1969. A new statistical approach to geographic variation analysis. Systematic Zoology 18:259-278.

HAMRICK, J.L. 1990. Isozymes and the analysis of genetic structure in plant populations. In Isozymes in plant biology (D.E. Soltis \& P.S. Soltis, eds.). Chapman and Hall, London, p.87-105.

HAMRICK, J.L. \& GODT, M.J.W. 1989. Allozyme diversity in plant species. In Plant population genetics, breeding, and germplasm resources (A.H.D. Brown, M.T. Clegg, A.L. Kahler \& B.S. Weir, eds.). Sinauer Associates, Sunderland, p.43-63.

HEYWOOD, J.S. 1991. Spatial analysis of genetic variation in plant populations. Annual Review of Ecology and Systematics 22:335-355.

KNOWLES, P., PERRY, D.J. \& FOSTER, H.A. 1992. Spatial genetic structure in two Tamarack [Larix laricina $(\mathrm{Du}$ Roi) K. Koch] populations with differing establishment histories. Evolution 46:572-576.

LEGENDRE, P. 1993. Spatial autocorrelation: trouble or new paradigm? Ecology 74:1659-1673.

LEGENDRE, P. \& FORTIN, M.J. 1989. Spatial pattern and ecological analysis. Vegetatio 80:107-138. 
LEGENDRE, P., ODEN, N.L., SOKAL, R.R., VAUDOR, A. \& KIM, J. 1990. Approximate analysis of variance of spatially autocorrelated regional data. Journal of Classification 7:53-75.

LEVIN, D.A. \& KERSTER, H.W. 1974. Gene flow in seed plants. Evolutionary Biology 7:139-220.

LEVINS, R. 1968. Evolution in changing environments. Princeton University Press, Princeton.

LINHART, Y.B., MITTON, J.B., STURGEON, K.B. \& DAVIS, M.L. 1981. Genetic variation in space and time in a population of ponderosa pine. Heredity 46:407-426.

LOVELESS, M.D. \& HAMRICK, J.L. 1984. Ecological determinants of genetic structure in plant populations. Annual Review of Ecology and Systematics 15:65-95.

LUNDKVIST, K. 1982. Genetic structure in natural and cultivated forest tree populations. Silva Fennica 16:141-149.

MORAES, P.L.R. 1997. Estrutura genética de populações de Cryptocarya moschata Nees \& Martius ex Nees (Lauraceae). Tese de doutorado, Universidade Estadual Paulista "Júlio de Mesquita Filho", Rio Claro.

MORAES, P.L.R. \& MONTEIRO, R. 2002. Taxas de cruzamento em uma população natural de Cryptocarya moschata Nees (Lauraceae). Biota Neotropica 2. http:// www.biotaneotropica.cria.org.br/v $2 \mathrm{n} 2 / \mathrm{pt} /$ fullpaper?bn01102022002+pt (acesso em 07/08/2002).

MORAES, P.L.R. \& PAOLI, A.A.S. 1995. Dispersão e germinação de sementes de Cryptocarya moschata Nees \& Martius ex Nees, Ocotea catharinensis Mez e Endlicheria paniculata (Sprengel) MacBride (Lauraceae). Arquivos de Biologia e Tecnologia 38:1119-1129.

MORAES, P.L.R. \& PAOLI, A.A.S. 1999. Morfologia e estabelecimento de plântulas de Cryptocarya moschata Nees, Ocotea catharinensis Mez e Endlicheria paniculata (Spreng.) MacBride - Lauraceae. Revista Brasileira de Botânica 22:187-295.

MORAES, P.L.R., MONTEIRO, R. \& VENCOVSKY, R. 1999. Conservação genética de populações de Cryptocarya moschata Nees (Lauraceae) na Mata Atlântica do estado de São Paulo. Revista Brasileira de Botânica 22:237-248.

MORAES, P.L.R., MONTEIRO, R. \& VENCOVSKY, R. 2002. Genetic differentiation and diversity of natural populations of Cryptocarya spp. (Lauraceae) from the Brazilian Atlantic rain forest. Lundiana 3:99-109.

PEAKALL, R. \& SMOUSE, P.E. 2001. GenAlEx V5: Genetic Analysis in Excel. Population genetic software for teaching and research. Australian National University, Canberra, Australia. http://www.anu.edu.au/BoZo/ GenAlEx/ (acesso em 12/2001).

PEAKALL, R., RUIBAL, M. \& LINDENMAYER, D.B. 2003. Spatial autocorrelation analysis offers new insights into gene flow in the australian bush rat, Rattus fuscipes. Evolution 57:1182-1195.

PERRY, D.J. \& KNOWLES, P. 1991. Spatial genetic structure within three sugar maple (Acer saccharum Marsh.) stands. Heredity 66:137-142.
SAKAI, A.K. \& ODEN, N.L. 1983. Spatial patterns of sex expression in silver mapple (Acer saccharinum L.): Morisita's index and spatial autocorrelation. The American Naturalist 122:489-508.

SHEA, K.L. \& FURNIER, G.R. 2002. Genetic variation and population structure in central and isolated populations of Balsam Fir, Abies balsamea (Pinaceae). American Journal of Botany 89:783-791.

SCHOEN, D.J. \& LATTA, R.G. 1989. Spatial autocorrelation of genotypes in populations of Impatiens pallida and Impatiens capensis. Heredity 63:181-190.

SHAPCOTT, A. 1995. The spatial genetic structure in natural populations of the Australian temperate rainforest tree Atherosperma moschatum (Labill.) (Monimiaceae). Heredity 74:28-38.

SLATKIN, M. \& ARTER, H.E. 1991. Spatial autocorrelation methods in population genetics. The American Naturalist 138:499-517.

SMOUSE, P.E. \& PEAKALL, R. 1999. Spatial autocorrelation analysis of individual multiallele and multilocus genetic structure. Heredity 82:561-573.

SOKAL, R.R. 1979. Ecological parameters inferred from spatial autocorrelograms. In Contemporary quantitative ecology and related econometrics (G.P. Patil \& M.L. Rosenzweig, eds.). International Cooperative Publishing House, Fairland, p.167-196.

SOKAL, R.R. \& ODEN, N.L. 1978a. Spatial autocorrelation in biology. 1. Methodology. Biological Journal of the Linnaean Society 10:199-228.

SOKAL, R.R. \& ODEN, N.L. 1978b. Spatial autocorrelation in biology. 2. Some biological implications and four applications of evolutionary and ecological interest. Biological Journal of the Linnaean Society 10:229-249.

SOKAL, R.R., JACQUEZ, G.M. \& WOOTEN, M.C. 1989. Spatial autocorrelation analysis of migration and selection. Genetics 121:845-855.

SOUTHWOOD, T.R.E. 1987. The concept and nature of the community. In Organization of communities: past and present (J.H.R. Gee \& P.S. Giller, eds.). Blackwell Scientific Publications, Oxford, p.3-27.

TELLES, M.P.C., DINIZ FILHO, J.A., COELHO, A.S.G. \& CHAVES, L.J. 2001. Autocorrelação espacial das freqüências alélicas em subpopulações de cagaiteira (Eugenia dysenterica DC., Myrtaceae) no sudeste de Goiás. Revista Brasileira de Botânica 24:145-154.

TOKUNAGA, T. \& OHNISHI, O. 1992. Spatial autocorrelation analysis of allozyme variants within local sites of wild radish population. Japanese Journal of Genetics 67:209-216.

WARTENBERG, D. 1989. SAAP: spatial autocorrelation analysis program. Exeter Publishers, New York.

WRIGHT, S. 1943. Isolation by distance. Genetics 28:114-138.

YEH, F.C.H. 1989. Isozyme analysis for revealing population structure for use in breeding strategies. In Proceedings of the Conference on Breeding tropical trees: population structure and genetic improvement strategies in clonal and seedling forestry (G.I. Gibson, A.R. Griffin \& A.C. Matheson, eds.). Oxford Forestry Institute, Winrock International, Oxford, Arlington, p.119-131. 